\title{
The role of processing and the food matrix in allergenicity of foods
}

\author{
Mills E.N.C, Sancho A.I., Rigby N., Jenkins J.A., Mackie A.R. \\ Institute of Food Research, \\ Norwich Research Park, \\ Colney Norwich, \\ NR4 7UA \\ UK \\ Clare.mills@bbsrc.ac.uk
}

\begin{abstract}
The agents that cause IgE-mediated allergies are known as allergens and are almost always proteins. It is emerging that the way in which food proteins elicit an allergic reaction can be modified by food processing procedures. This is because food processing alters the structure of food proteins through either unfolding and aggregation, or covalent modification by other food components such as sugars. In this way the IgE-recognition sites on an allergen, known as epitopes, can either be destroyed or new epitopes formed. Processing can destroy the allergenicity of some proteins, notably the Bet $\mathrm{v} 1$ homologues, which both unfold and become modified with plant polyphenols. Others, such as the prolamin superfamily members, the nsLTP and $2 \mathrm{~S}$ albumin allergens, have stable protein scaffolds and either do not unfold or re-fold on cooling, retaining their allergenicity. Some allergens are highly thermostable because of their mobile structures which are not disrupted on heating, such as the caseins and seed storage prolamins of wheat. Others, such as seed storage globulins, only partially-unfold and can retain much of their allergenicity. The structure of the food matrix may also affect the release and stability of allergens impacting on the elicitation of reactions in food allergic individuals. Such complexity makes it a challenge to develop generic food processing procedures capable of removing or reducing allergenicity which are effective for all allergic consumers. A better knowledge of how processing affects the allergenicity of food is also important for risk assessors and managers involved in managing allergenic hazards in food.
\end{abstract}

Key words: Food, allergy, processing, protein structure 


\section{Introduction}

Food allergies are generally held to be adverse reactions to foods which have an immunological basis. They include both IgE-mediated allergies usually classified as Type I hypersensitivity reactions and the gluten intolerance syndrome, Coeliac's disease. With regards the former, IgE is produced as part of the normal functioning of the immune system in response to parasitic infections. For reasons that are not fully understood some individuals begin to make IgE to various environmental agents, including dusts, pollens and foods. IgEmediated allergies develop in two phases, (1) sensitisation when IgE production is stimulated and (2) elicitation when an individuals experiences and adverse reaction on re-exposure to an allergen. Both stages are triggered by allergens which are almost always proteins. In an allergic reaction allergen is recognised by $\operatorname{IgE}$ bound to the surface of histamine containing mast cells, cross-linking the $\mathrm{IgE}$ in the process and triggering the release of inflammatory mediators such as histamine. It is these mediators that cause the acute inflammatory reactions which become manifested as respiratory (asthma, rhinitis), cutaneous (eczema, urticaria) or gastrointestinal (vomiting, diarrhoea) symptoms which may occur alone or in combination in an allergic reaction. A rare but very severe reaction is anaphylactic shock characterised by respiratory symptoms, fainting, itching, urticaria, swelling of the throat or other mucous membranes, and a dramatic loss of blood pressure.

In contrast the gluten intolerance syndrome, Coeliac's disease, is manifested in a much slower manner than IgE-mediated allergies an individual taking hours or days to react. Thought to affect around $1 \%$ of the population it afflicts more women than men and arises as a consequence of deamidation of the glutamine residues in gluten peptides by the gut mucosal transglutaminase. The modified peptides are able to bind to class II human histocompatibility leucocyte antigen (HLA) molecules DQ2 and DQ8. This recognition event appears to orchestrate an abnormal cellular-mediated immune response which triggers an inflammatory reaction which results in the flattened mucosa characteristic of Coeliac's disease (Hischenhuber et al., 2006).

Two of the major questions in food allergy research, particularly in relation to IgE-mediated allergies are what makes one person, and not another, become allergic and what are the attributes of some foods and food proteins so much more allergenic than others? Seeking answers to these questions is more difficult in relation to food allergies than inhalant allergies, partly because we lack effective animal models for oral sensitisation. Many animal models requiring co-administration of adjuvants such as cholera toxin or polysaccharides such as carageenen, before an IgE response can be elicited (Knippels and Penninks 2003). It is also made more complex because the of the way in which food proteins involved in sensitising or eliciting allergic reactions are altered by food processing procedures. For example they often become an insoluble mass which is not amenable to extraction in the simple salt solutions routinely employed for serological or clinical analyses. Furthermore the structure of a food itself may affect the way in which allergens are broken down during digestion and presented to the immune system during both the sensitisation and elicitation phases of allergic responses. It has been shown that the structure of the food matrix can affect the elicitation of allergic reactions in food allergic individuals, fat-rich matrices such as chocolate, affecting the kinetics of allergen release, potentiating the severity of allergic reactions (Grimshaw et al., 2003).

As there is no cure for either IgE-mediated allergies or Coeliac's disease individuals with these conditions have to exclude problems foods, together with any derived ingredients, from 
their diet. Consequently food labelling legislation has been modified around the world in response to the Codex Alimentarius Commission amendment to the Codex General Standard for the Labelling of Prepackaged Foods (Codex General Standard for the Labelling of Prepackaged Foods (1999) CODEX STAN 1-1985 (Rev. 1-1991)) which aims to ensure that allergic consumers have good quality information on pre-packaged foods about their content of allergens. In addition any risk assessment of a novel food (including GMOs) or novel food processing method has to include an explicit allergenic risk assessment. Understanding how food processing affects allergenic potential is therefore important if allergenic risks are to be managed effectively and minimise the risks of adverse reactions to foods. Since we do not understand the molecular events involved in an individual becoming sensitised to a particular protein, and we have no adequate animal models for food allergy, it is difficult to study how food processing can affect the ability of an allergen or a type of food to sensitise an individual. Consequently this paper is focussed on our knowledge of how processing affects elicitation of IgE-mediated allergic reactions to food.

\section{Allergens and epitopes in IgE-mediated allergies}

The World Health Organisation and the International Union of Immunological Societies produces an official list of allergens, which are designated by the Allergen Nomenclature sub-committee. Allergens included in this listing must induce IgE-mediated (atopic) allergy in humans with a prevalence of IgE reactivity above 5\%. An allergen is termed major if it is recognised by IgE from at least $50 \%$ of a cohort of allergic individuals but does not carry any connotation of allergenic potency; allergens are otherwise termed "minor". The allergen designation is then based on the Latin name of the species from which it originates and is composed of the first three letters of the genus, followed by the first letter of the species finishing with an arabic number e.g. Ara $\mathrm{h} 1$ relates to an allergen from Arachis hypogea (peanuts).

The sites on a protein which are recognised by an antibody are known as epitopes and can be either linear or conformational (Van Regenmortel M.H.V. 1992). In the former only the primary sequence of a polypeptide is involved in antibody recognition, but in conformational epitopes the three-dimensional structure of a protein is also important. Thus the latter are formed from a number of segments of the polypeptide chain which may be quite distant in the amino acid sequence of a protein but are brought together spatially as a consequence of its tertiary and quaternary structure. Most epitopes are thought to be conformational in nature and are particularly difficult to define in relation to food allergens where processing can have such a disruptive effect on native protein structure. The ability of an allergen to elicit a reaction can be modified by disrupting the IgE epitopes. It is also possible that food processing could introduce new epitopes, sometimes termed neo-epitopes. This may occur through protein unfolding revealing inner portions of a proteins structure not generally available for antibody binding or the covalent modification of a protein by sugars or other food components.

\section{Processing-labile allergens}

The Bet v 1 superfamily of plant food allergens, which are involved in the pollenfruit/vegetable cross-reactivity syndrome are generally unstable to common food processing technologies. This is probably because the main cross-reactive $\operatorname{IgE}$ binding sites on these proteins (also known as epitopes) are primarily directed towards conformational epitopes (Neudecker et al., 2001;Gajhede et al., 1996). Bet v 1 proteins lack the stabilising effect of intramolecular disulphide bonds and as a result unfold on heating, disrupting conformational epitopes. Thus, as a general rule both the IgE-reactivity and ability of Bet v 1 homologues to 
trigger a reaction in sensitized individuals is reduced by food processing. However, the extent to which they unfold may depend on the form of food processing. Thus, heating in low-water systems, such as roasting of hazelnuts, whilst reducing the allergenicity of Bet $\mathrm{v} 1$ homologues, did not abolish it for all patients (Hansen et al., 2003). In contrast cooking of fruits, such as apple, which have a high water activity, reduces their allergenicity in birchpollen allergic individuals. These observations can be explained by the general observation that protein thermostability is dependent on water activity (Gekko and Timasheff 1981).

\section{Processing stable allergens}

Allergens that do not loose their IgE-epitopes following processing generally fall into one of three different categories, the properties and characteristics which are summarised below.

Thermostable proteins which refold on cooling: depending on the conditions of heating many of the protein scaffolds characteristic of food allergens either resist thermal denaturation and/or are able to refold on cooling to the native folded conformation. One group of proteins that belong to this group are members of the prolamin superfamily. These proteins are characterized by a conserved pattern of cysteine residues, with either six or eight such residues forming either three or four intra-chain disulphide bonds which constrain the folded structure of the proteins. Consequently they resist thermal denaturation and may refold to the original native conformation. In general the allergenic properties of these proteins is retained following processing as has been found for the ns LTP allergens which retain their allergenic properties after heating including cooking of foods such as polenta (Pastorello et al., 2000) or fermentation to produce beer (Asero et al., 2001) or wine (Pastorello et al., 2003).

Inherently mobile proteins: There are a number of proteins which, instead of adopting compact globular structures, appear to comprise regions of ill-defined, disordered, mobile structures (Dunker et al., 2001). Such structures are dynamic, possessing a range of rapidly interchanging conformations and consequently do not show the co-operative transition on heating characteristic of a globular protein as it moves from a folded to an unfolded or partially folded structure. This class of proteins is represented by two examples of food allergens, caseins from milk and the prolamin seed storage proteins from cereals. The absence of a co-operative transition for caseins as determined by DSC and their lack of secondary structure has led to them being termed rheomorphic, rheo meaning to flow, and morphe meaning shape, a property shared by seed storage prolamins (Paulsson and Dejmek 1990; Holt and Sawyer 1993) As a consequence of their dynamic nature, these proteins possess many linear, and hence potentially thermo-stable, IgE epitopes and is probably the reason why the $\mathrm{IgE}$ binding capacity of caseins and wheat prolamins is largely unaltered by thermal processing (Simonato et al., 2001;Kohno et al., 1994) .

Proteins which only partially unfold: Many food proteins unfold to some extend during processing and the partially-unfolded proteins then interacting to form aggregates or aggregated protein networks such as those found in heat-set gels or interfacial layers in foams and emulsions. This group probably represents the majority of globular food proteins and include, for example the cow's milk whey proteins $\beta$-lactoglobulin and $\alpha$-lactalbumin, or the seed storage globulins such as the $11 \mathrm{~S}$ and $7 \mathrm{~S}$ seed storage globulins of soya (Mills et al., 2002).

Both 11S and 7S globulins, in common with other members of the cupin superfamily, are thermo-stabile proteins, the 7S globulins having their major thermal transition at around 70- 
$75^{\circ} \mathrm{C}$, whilst $11 \mathrm{~S}$ globulins unfold at temperatures above $94^{\circ} \mathrm{C}$, as determined by differential scanning calorimetry. However, whilst these proteins form large aggregates following heating and unfolding of the glycinin structure is irreversible, this is accompanied by little change in the native-like -sheet structures (Mills et al., 2003). Such observations suggest that some local unfolding occurs following heating, that the -sheet structure of the cupin fold is largely unaltered. Process such as adsorption to interfaces can also cause proteins to unfold and form aggregated networks (Mackie et al., 2000). However, indications are that this process also results in only limited unfolding in several protein systems, including the whey protein $\beta$-Lactoglobulin ( $\beta$-Lg) (Husband et al., 2001) or plant seed proteins such as the $2 \mathrm{~S}$ albumins (Burnett et al., 2002) and $\alpha$-amylase inhibitors of cereals (Gilbert et al., 2003).

\section{Processing induced chemical modification of allergens}

The reaction between free amino groups on proteins and the aldehyde or ketone groups of sugars known as Maillard's reaction is one of the major chemical reactions that take's place in foods during processing. As a result of these non-enzymatic glycation reactions, food proteins can become modified in a complex, diverse way with Amadori products or advanced glycation end products $\left(\mathrm{AGE}_{\mathrm{S}}\right)$. The Maillard adduct rearrangement products can also crosslink food proteins, and in studies on the IgE-reactivity of bread in a panel of wheat allergic individuals suggested that some of the IgE-reactive protein was extensively cross-linked by Maillard adducts (Simonato et al., 2001). This cross-linking ability may enhance the IgEbinding capacity of allergens, and indications are that some, such as Ara $\mathrm{h} 1$ and Ara $\mathrm{h} 2$ from peanut, are able to form high molecular weight aggregates which bind IgE more effectively than unmodified allergens (Maleki et al., 2000;Chung and Champagne 2001). Such results are consistent with the observation that the serum IgE-binding in peanut allergic individuals was greater to roasted, with boiled or fried peanuts (Beyer et al., 2001). These data indicate that certain types of thermal processing can introduce additional IgE binding sites. However, these observations may be complicated by the fact that peanut allergens leach out of peanuts during boiling, lowering the residual allergen content in the boiled nuts (Mondoulet et al., 2005). Maillard modification has also been found to increase the IgE-binding capacity of the allergenic shellfish tropomyosin (Nakamura et al., 2005). Individuals may have become sensitised to glycated tropomyosin itself, through consumption of the dried fish products frequently used, especially in oriental cuisine.

In contrast to the observation with peanutsand shellfish, glycation of fruit allergens does not appear to increase their allergenicity. For example, glycation of Pru av 1, the allergenic Bet v 1 homologue of cherry, significantly reduces its $\operatorname{IgE}$ reactivity, whilst modification with carbonyl compounds formed during carbohydrate breakdown, such as glyoxal and glycoaldehyde, almost completely abolished IgE binding (Gruber et al., 2005). In addition glycation of the nsLTP allergen from apple, Mal d 3, was also protected the IgE-binding capacity of the protein following harsh thermal treatment (Sancho et al., 2005).

Other types of processing-induced modification which may affect allergenicity include interactions with oxidsed lipids (Doke et al., 1989) and enzymatic modification with polyphenols catalysed by the polyphenol oxidase. Modification with epichatechin and caffeic acid was found to reduce the IgE-binding capacity of Pru av 1. However the extent to which it was reduced was highly dependent on the polyphenol involved, quercetin and quercetinglycoside, rutin, having a lesser effect (Gruber et al., 2004). Such enzymatic modifications may be responsible for the highly labile nature of many fruit Bet $\mathrm{v} 1$ type allergens. 


\section{Conclusion}

Whilst understanding the impact of food processing and food structure on allergenic potential is central to managing allergen risks in the food chain there do not appear to be any clear rules regarding how different allergens within the same food respond to food processing. In fruits and vegetables for example, the allergenicity members of the Bet $\mathrm{v} 1$ family of allergens found in fruits is generally destroyed by cooking. However, the allergenicity of another type of allergen belonging to the ns LTP family, is largely unaltered by food processing. As a consequence the efficacy of food processing in removing allergenicity is a function of the type of allergy an individual suffers from. Such complexity makes it a challenge to develop generic food processing procedures capable of removing or reducing allergenicty which are effective for all allergic consumers. Our lack of understanding of the impact of conventional food processing procedures also makes the assessment of novel processes, such as high pressure, or novel thermal processing procedures, less certain.

Two areas that remain neglected with regards investigating the impact food processing has on allergenicity relates to measures of sensitisation potential and the way in which it may alter thresholds for elicitation of allergic reactions in sensitised individuals. The latter is essential information for managing allergens in a factory environment, particularly in relation to crosscontact allergens, which find their way into foods otherwise free from them through parallel or common processing lines. It may be that certain types of food structure, for example fatcontinuous versus aqueous continuous matrices, may raise or lower the threshold doses for important allergens such as those from peanut. It is lso known that food processing can affect the responsiveness of the immunoassay methods used to monitor allergens in foods and equipment clean-down ((Poms et al., 2004). A better understanding of how processing affects allergen structure, and hence allergen screening assays, would help support interpretation of immunoassay results especially when used to monitor highly processed ingredients.

\section{Acknowledgements}

This work was supported by the competitive strategic grant to IFR from BBSRC and EU through EuroPrevall (CT-514000).

\section{Reference List}

Asero R., Mistrello G., Roncarolo D., Amato S. and van Ree R. (2001) A case of allergy to beer showing cross-reactivity between lipid transfer proteins. Ann.Allergy Asthma Immunol. $87,65-67$.

Beyer K., Morrow E., Li X.M., Bardina L., Bannon G.A., Burks A.W. and Sampson H.A. (2001) Effects of cooking methods on peanut allergenicity. J.Allergy Clin.Immunol. 107, 1077-1081.

Burnett G.R., Rigby N.M., Mills E.N.C., Belton P.S., Fido R.J., Tatham A.S. and Shewry P.R. (2002) Characterization of the emulsification properties of $2 \mathrm{~S}$ albumins from sunflower seed. Journal of Colloid and Interface Science 247, 177-185.

Chung S.Y. and Champagne E.T. (2001) Association of end-product adducts with increased IgE binding of roasted peanuts. J.Agric.Food Chem. 49, 3911-3916. 
Doke S., Nakamura R. and Torii S. (1989) Allergenicity of Food Proteins Interacted with Oxidized Lipids in Soybean-Sensitive Individuals. Agricultural and Biological Chemistry 53, 1231-1235.

Dunker A.K., Lawson J.D., Brown C.J., Williams R.M., Romero P., Oh J.S., Oldfield C.J., Campen A.M., Ratliff C.R., Hipps K.W., Ausio J., Nissen M.S., Reeves R., Kang C.H., Kissinger C.R., Bailey R.W., Griswold M.D., Chiu M., Garner E.C. and Obradovic Z. (2001) Intrinsically disordered protein. Journal of Molecular Graphics \& Modelling 19, 26-59.

Gajhede M., Osmark P., Poulsen F.M., Ipsen H., Larsen J.N., vanNeerven R.J.J., Schou C., Lowenstein H. and Spangfort M.D. (1996) X-ray and NMR structure of Bet v 1, the origin of birch pollen allergy. Nature Structural Biology 3, 1040-1045.

Gekko K. and Timasheff S.N. (1981) Mechanism of Protein Stabilization by Glycerol Preferential Hydration in Glycerol-Water Mixtures. Biochemistry 20, 4667-4676.

Gilbert S.M., Burnett G.R., Mills E.N.C., Belton P.S., Shewry P.R. and Tatham A.S. (2003) Identification of the wheat seed protein $\mathrm{CM} 3$ as a highly active emulsifier using a novel functional screen. Journal of Agricultural and Food Chemistry 51, 2019-2025.

Grimshaw K.E.C., King R.M., Nordlee J.A., Hefle S.L., Warner J.O. and Hourihane J.O.B. (2003) Presentation of allergen in different food preparations affects the nature of the allergic reaction - a case series. Clinical and Experimental Allergy 33, 1581-1585.

Gruber P., Becker W.M. and Hofmann T. (2005) Influence of the Maillard reaction on the allergenicity of rAra h 2, a recombinant major allergen from peanut (Arachis hypogaea), its major epitopes, and peanut agglutinin. Journal of Agricultural and Food Chemistry 53, 22892296.

Gruber P., Vieths S., Wangorsch A., Nerkamp J. and Hofmann T. (2004) Maillard reaction and enzymatic browning affect the allergenicity of Pru av 1, the major allergen from cherry (Prunus avium). Journal of Agricultural and Food Chemistry 52, 4002-4007.

Hansen K.S., Ballmer-Weber B.K., Luttkopf D., Skov P.S., Wuthrich B., Bindslev-Jensen C., Vieths S. and Poulsen L.K. (2003) Roasted hazelnuts - allergenic activity evaluated by double-blind, placebo-controlled food challenge. Allergy 58, 132-138.

Hischenhuber C., Crevel R., Jarry B., Maki M., Moneret-Vautrin D.A., Romano A., Troncone R. and Ward R. (2006) Review article: safe amounts of gluten for patients with wheat allergy or coeliac disease. Alimentary Pharmacology \& Therapeutics 23, 559-575.

Holt C. and Sawyer L. (1993) Caseins As Rheomorphic Proteins - Interpretation of Primary and Secondary Structures of the Alpha-S1-Caseins, Beta-Caseins and Kappa-Caseins. Journal of the Chemical Society-Faraday Transactions 89, 2683-2692.

Husband F.A., Garrood M.J., Mackie A.R., Burnett G.R. and Wilde P.J. (2001) Adsorbed protein secondary and tertiary structures by circular dichroism and infrared spectroscopy with refractive index matched emulsions. Journal of Agricultural and Food Chemistry 49, 859866. 
Knippels L.M. and Penninks A.H. (2003) Assessment of the allergic potential of food protein extracts and proteins on oral application using the brown Norway rat model. Environ.Health Perspect. 111, 233-238.

Kohno Y., Honma K., Saito K., Shimojo N., Tsunoo H., Kaminogawa S. and Niimi H. (1994) Preferential Recognition of Primary-Protein Structures of Alpha-Casein by Igg and Ige Antibodies of Patients with Milk Allergy. Annals of Allergy 73, 419-422.

Mackie A.R., Gunning A.P., Wilde P.J. and Morris V.J. (2000) Orogenic displacement of protein from the oil/water interface. Langmuir 16, 2242-2247.

Maleki S.J., Chung S.Y., Champagne E.T. and Raufman J.P. (2000) The effects of roasting on the allergenic properties of peanut proteins. J.Allergy Clin.Immunol. 106, 763-768.

Mills E.N., Jenkins J., Marigheto N., Belton P.S., Gunning A.P. and Morris V.J. (2002) Allergens of the cupin superfamily. Biochem.Soc.Trans. 30, 925-929.

Mills E.N.C., Marigheto N.A., Wellner N., Fairhurst S.A., Jenkins J.A., Mann R. and Belton P.S. (2003) Thermally induced structural changes in glycinin, the 11S globulin of soya bean (Glycine max) - an in situ spectroscopic study. Biochimica et Biophysica Acta-Proteins and Proteomics 1648, 105-114.

Mondoulet L., Paty E., Drumare M.F., Ah-Leung S., Scheinmann P., Willemot R.M., Wal J.M. and Bernard H. (2005) Influence of thermal processing on the allergenicity of peanut proteins. Journal of Agricultural and Food Chemistry 53, 4547-4553.

Nakamura A., Watanabe K., Ojima T., Ahn D.H. and Saeki H. (2005) Effect of Maillard reaction on allergenicity of scallop tropomyosin. Journal of Agricultural and Food Chemistry $53,7559-7564$.

Neudecker P., Schweimer K., Nerkamp J., Scheurer S., Vieths S., Sticht H. and Rosch P. (2001) Allergic cross-reactivity made visible: solution structure of the major cherry allergen Pru av 1. J.Biol.Chem. 276, 22756-22763.

Pastorello E.A., Farioli L., Pravettoni V., Ispano M., Scibola E., Trambaioli C., Giuffrida M.G., Ansaloni R., Godovac-Zimmermann J., Conti A., Fortunato D. and Ortolani C. (2000) The maize major allergen, which is responsible for food-induced allergic reactions, is a lipid transfer protein. J.Allergy Clin.Immunol. 106, 744-751.

Pastorello E.A., Farioli L., Pravettoni V., Ortolani C., Fortunato D., Giuffrida M.G., Garoffo L.P., Calamari A.M., Brenna O. and Conti A. (2003) Identification of grape and wine allergens as an endochitinase 4, a lipid-transfer protein, and a thaumatin. Journal of Allergy and Clinical Immunology 111, 350-359.

Paulsson M. and Dejmek P. (1990) Thermal-Denaturation of Whey Proteins in Mixtures with Caseins Studied by Differential Scanning Calorimetry. Journal of Dairy Science 73, 590-600.

Poms R.E., Klein C.L. and Anklam E. (2004) Methods for allergen analysis in food: a review. Food Additives and Contaminants 21, 1-31.

Sancho A.I., Rigby N.M., Zuidmeer L., Asero R., Mistrello G., Amato S., Gonzalez-Mancebo E., Fernandez-Rivas M., Ree R. and Mills E.N.C. (2005) The effect of thermal processing on 
the IgE reactivity of the non-specific lipid transfer protein from apple, Mal d 3. Allergy 60, 1262-1268.

Simonato B., Pasini G., Giannattasio M., Peruffo A.D., De Lazzari F. and Curioni A. (2001) Food allergy to wheat products: the effect of bread baking and in vitro digestion on wheat allergenic proteins. A study with bread dough, crumb, and crust. J.Agric.Food Chem. 49, $5668-5673$.

Van Regenmortel M.H.V. (1992) Molecular dissection of protein antigens. In . Ed.. In Structure of Antigens Vol 1 (Edited by Van Regenmortel M.H.V.), pp. 1-28. CRC Press Inc.. 